\title{
Uso de mioinositol más Bifidobacterium lactis y Lactobacillus rhamnosus para la prevención de diabetes mellitus gestacional en mujeres mexicanas
}

\author{
Enrique Reyes-Muñoz ${ }^{*}$, Salvador Espino y Sosa², Claudia M. Flores-Robles ${ }^{3}$, \\ Lidia Arce-Sánchez ${ }^{3}$, Nayeli Martínez-Cruz³, Guillermo Garduño-García ${ }^{3}$, \\ Cesar R. Tawney-Serrano ${ }^{4}$, Juan J. Domínguez-Rodríguezº, María L. Martínez-Hernández \\ Lilia R. Pérez-Mota7, Lourdes C. Llanes-Carrillo ${ }^{8}$ y Marcelo González-Rodríguez ${ }^{9}$ \\ ${ }^{1}$ Coordinación de Endocrinología Ginecológica y Perinatal, Instituto Nacional de Perinatología Isidro Espinosa de los Reyes, Ciudad de México, \\ Méx.; ${ }^{2}$ Subdirección de Investigación clínica, Instituto Nacional de Perinatología Isidro Espinosa de los Reyes, Ciudad de México, Méx.; ${ }^{3}$ Coordinación \\ de Endocrinología, Instituto Nacional de Perinatología Isidro Espinosa de los Reyes, Ciudad de México, Méx.; ${ }^{4}$ División de Ginecología y Obstetricia, \\ Hospital General de Occidente, Zapopan, Jal.; ${ }^{5}$ Clínica Médica la Quinta, Matamoros, Tamps.; ${ }^{6}$ Centro de Colposcopia de Aguascalientes, \\ Aguascalientes, Ags.; ${ }^{7}$ Sanatorio y Clínica Campeche, Campeche, Camp.; ${ }^{8}$ Consultorio Privado, Campeche, Camp.; ${ }^{9}$ Servicio de Ginecología y \\ Obstetricia, Hospital Faro del Mayab, Mérida, Yuc. México
}

\section{Resumen}

Objetivo: Comparar la incidencia de diabetes mellitus gestacional (DMG) en mujeres con tres o más factores de riesgo para desarrollar DMG suplementadas con mioinositol más probióticos versus mujeres sin suplementación. Material y métodos: Estudio de cohorte retrospectivo, grupo 1, mujeres con suplementación (mioinositol $2 \mathrm{~g}$ más Bifidobacterium lactis y Lactobacillus rhamnosus $5 \times 10^{8}$ unidades formadoras de colonias, dos veces al día, de las 12-14 hasta las 28 semanas de gestación); grupo 2, mujeres con control prenatal habitual sin suplementación, pareadas por edad e índice de masa corporal (IMC). El resultado primario fue la incidencia de DMG utilizando los criterios de la Asociación Internacional de Grupos de Estudio de Diabetes y Embarazo. Resultados: Grupo 1, $n=48$, y grupo $2 n=96$. No hubo diferencias significativas en características basales como edad, IMC, y numero de factores de riesgo entre los grupos. La incidencia de DMG en el grupo 1 fue $n=14$ (29.2\%) y en el grupo $2 n=46$ (47.9\%); RR: 0.61 (IC 95\%: 0.37-0.99; $p=0.03$ ). Conclusiones: La suplementación desde las 12-14 semanas de gestación con mioinositol más probióticos disminuye la incidencia de DMG en mujeres mexicanas.

PALABRAS CLAVE: Diabetes mellitus gestacional. Mioinositol. Probióticos. Embarazo.

\section{Use of myo-inositol plus Bifidobacterium lactis and Lactobacillus rhamnosus for preventing gestational diabetes mellitus in Mexican women}

\section{Abstract}

Aim: To compare the incidence of gestational diabetes mellitus (GDM) in women with three or more risk factor to developing GDM supplemented with myo-inositol plus probiotics versus women care without supplementation. Methods: Retrospective cohort study, group 1, women with supplementation (myo-inositol $2 \mathrm{~g}$ plus Bifidobacterium lactis and Lactobacillus rhamnosus $5 \times 108$ UFC, twice per day, from 12-14 to 28 weeks of gestation; group 2, women with prenatal care without supplementation,

Correspondencia:

*Enrique Reyes-Muñoz

E-mail: dr.enriquereyes@gmail.com
Gac Med Mex. 2020;156(Supl 3): S51-S57

Disponible en PubMed

www.gacetamedicademexico.com

0016-3813/๑ 2020 Academia Nacional de Medicina de México, A.C. Publicado por Permanyer. Este es un artículo open access bajo la licencia CC BY-NC-ND (http://creativecommons.org/licenses/by-nc-nd/4.0/). 
matched by age and body mass index (BMI). The primary outcome was the incidence of GDM using the International Association of Diabetes and Pregnancy Study Groups criteria. Results: Group $1 n=48$, group $2 n=96$. There were no significant baseline differences between groups in age, BMI and number of risk factors. The incidence of GDM in group 1 was $n=14$ (29.2\%), and for group $2 n=46$ (47.9\%); RR: 0.61 (95\% Cl: 0.37-0.99; $p=0.03$ ). Conclusions: Supplementation from 12-14 weeks of gestation with myo-inositol plus probiotics decrease the incidence of GDM in Mexican women.

KEY WORDS: Gestational diabetes mellitus. Myo-inositol. Probiotics. Pregnancy.

\section{Introducción}

La diabetes mellitus gestacional (DMG) se define como aquella diabetes diagnosticada en el segundo o tercer trimestre del embarazo y que claramente no es una diabetes manifiesta'. La prevalencia de DMG varía ampliamente dependiendo de la población, si se realiza la estrategia en un paso $o$ en dos pasos y los criterios diagnósticos utilizados ${ }^{2,3}$. La Federación Internacional de Diabetes ha estimado en el año 2019 una prevalencia mundial de diabetes en el embarazo del $15.8 \%{ }^{4}$ y para América del Norte y el Caribe (incluido México) una prevalencia del 20.1\%. En una encuesta reciente, realizada entre médicos que atienden embarazadas en cuatro ciudades de México (Mérida, Monterrey, Guadalajara y Ciudad de México), se reportó una prevalencia de DMG del $23.7 \% \%^{4,5}$.

La DMG se ha asociado con incremento en el riesgo de complicaciones maternas como: preeclampsia, cesárea $^{6,7}$ y a largo plazo el desarrollo de diabetes mellitus tipo 2 y enfermedad cardiovascular ${ }^{8}$, así como mayor riesgo de complicaciones fetales (entre ellas, macrosomía, distocia de hombros, neonatos grandes para edad gestacional, hipoglucemia neonatal ${ }^{6,7}$ y a largo plazo, obesidad, diabetes y enfermedad cardiovascular) ${ }^{8}$.

La DMG es el resultado de la disfunción de las células $\beta$ en un contexto de resistencia crónica a la insulina. Durante el embarazo el deterioro de las células $\beta$ aunado a la resistencia a la insulina inducida por el embarazo son componentes clave en la fisiopatología de la $\mathrm{DMG}^{8,9}$. En la mayoría de los casos, estas deficiencias existen antes del embarazo y pueden ser progresivas, lo que representa un mayor riesgo de DMT2 después del embarazo ${ }^{8,9}$.

Los factores de riesgo para la DMG están asociados directa o indirectamente con células $\beta$ deterioradas en su función y/o sensibilidad a la insulina alterada $^{8}$ e incluyen: sobrepeso, obesidad, ganancia excesiva de peso gestacional, grupo étnico latino, asiático o afroamericano, antecedentes personales de DMG, macrosomía, hipertensión arterial crónica, síndrome de ovarios poliquísticos, prediabetes y dislipidemia (colesterol vinculado a lipoproteínas de alta densidad [C-HDL] $<35 \mathrm{mg} / \mathrm{dl}$ y/o triglicéridos $>250 \mathrm{mg} / \mathrm{dl})^{8,10}$.

El mioinositol es uno de los nueve estereoisómeros del inositol, que es un precursor de fosfoinosítidos, que están involucrados en la traducción de la señalización celular y como segundos mensajeros con un efecto similar a la insulina, lo que se traduce en un efecto sensibilizador a la insulina ${ }^{11,12}$. En una revisión sistemática reciente que incluyó cinco estudios clínicos aleatorizados (ECA) se concluyó que la suplementación con mioinositol $4 \mathrm{~g}$ al día disminuye la incidencia de DMG, particularmente en mujeres con sobrepeso, obesidad, glucosa de ayuno entre 92 y $125 \mathrm{mg} / \mathrm{dl}$ y mujeres con antecedente familiar en primer grado con diabetes mellitus tipo $2^{13}$.

Los probióticos son microorganismos vivos y viables capaces de proporcionar beneficios específicos para la salud de su huésped, cuando se ingieren en cantidades adecuadas. Algunos estudios han vinculado los cambios en el microbioma intestinal durante el embarazo con el aumento de la resistencia a la insulina, así como el estado de inflamación y el estrés oxidativo $^{13,14}$. La suplementación con probióticos como Bifidobacterium lactis y Lactobacillus rhamnosus durante el embarazo sugiere que disminuyen la incidencia de DMG ${ }^{15,16}$.

A la fecha no hay estudios que reporten el efecto de la combinación de mioinositol más probióticos sobre la incidencia de DMG. El objetivo del presente estudio es comparar la incidencia de DMG en mujeres con alto riesgo de desarrollar DMG suplementadas con mioinositol $2 \mathrm{~g}$ más probióticos (B. lactis y L. rhamnosus $5 \times 10^{8}$ unidades formadoras de colonias [UFC]) vs. mujeres con control prenatal habitual sin suplementación.

\section{Material y métodos}

\section{Participantes}

Estudio de cohorte histórica en el que se incluyeron mujeres con embarazo único, con tres o más factores 
de riesgo para el desarrollo de DMG, entre ellos: sobrepeso, obesidad, sedentarismo, dislipidemia (c-HDL < $35 \mathrm{mg} / \mathrm{dl}$ o triglicéridos > $250 \mathrm{mg} / \mathrm{dl}$ ), grupo étnico latino, y alguno de los siguientes antecedentes: familiar en primer grado de diabetes mellitus tipo 2, DMG, recién nacido macrosómico (peso $>4 \mathrm{~kg}$ ), prediabetes (glucosa de ayuno entre 100 y $125 \mathrm{y} / \mathrm{o} \mathrm{hemog-}$ lobina glucosilada entre 5.7 y $6.4 \%$ ), hipertensión arterial crónica y/o síndrome de ovarios poliquísticos. En el grupo 1 las mujeres recibieron suplementación con mioinositol $2 \mathrm{~g}$ más $B$. lactis y $L$. rhamnosus $5 \times 10^{8}$ UFC (G-BALANCE ${ }^{\circledR}$, Nestlé), un sobre dos veces al día vía oral, desde las 12-14 hasta las 28 semanas de gestación (SDG). El grupo 2 estuvo compuesto por mujeres con control prenatal habitual sin suplementación con mioinositol y probióticos. Todas las mujeres recibieron ácido fólico más suplementos de hierro y/o multivitamínicos. Se excluyeron mujeres con diabetes mellitus pregestacional tipo 10 tipo 2, en tratamiento con corticosteroides, y con enfermedades autoinmunes, cardiopatías o nefropatías.

\section{Recolección de datos}

Se invitó a médicos con práctica privada o pública para enviar datos de pacientes que cumplían con los siguientes criterios de inclusión: mujeres con tres o más factores de riesgo para desarrollar DMG, con una curva de tolerancia oral a la glucosa (CTOG) $75 \mathrm{~g}-2$ $\mathrm{h}$ antes de las 15 SDG para descartar diabetes mellitus pregestacional, que recibieron suplementación con mioinositol más probióticos (G-BALANCE ${ }^{\circledR}$ ) un sobre cada 12 horas vía oral, desde las 12-14 hasta las $28 \mathrm{SDG}$, con una adherencia documentada de al menos el $90 \%$, que tuvieron una segunda CTOG 75 g-2 h entre las 22 y 30 SDG. Se les solicitó foto de ambas CTOG. Los casos recibidos llevaron el control prenatal en el año 2019. El grupo 2 se integró con mujeres que acudieron a control prenatal al Instituto Nacional de Perinatología (INPer) entre 2010 y 2011, todas tenían registrado los factores de riesgo, el IMC pregestacional, una CTOG $75 \mathrm{~g}-2 \mathrm{~h}$ realizada entre las 22 y 30 SDG, determinación de hemoglobina glucosilada al mismo tiempo que la CTOG, y no recibieron suplementación con mioinositol y probióticos durante la gestación. Los datos fueron obtenidos de una base de datos histórica, inicialmente se seleccionaron a mujeres con tres o más factores de riesgo para desarrollar DMG, que fueron pareadas de acuerdo con su edad e IMC, en proporción 2:1 con el grupo 1 , antes de conocer el resultado de la CTOG.

\section{Variable de resultado}

La variable de desenlace primario fue la incidencia de DMG. La DMG fue diagnosticada de acuerdo con los criterios de la Asociación Americana de Diabetes $(A D A)^{1}$, se consideró DMG con uno o más valores alterados en la CTOG $75 \mathrm{~g}-2 \mathrm{~h}$. Ayuno mayor o igual a $92 \mathrm{mg} / \mathrm{dl}, 1$ hora mayor o igual a $180 \mathrm{mg} / \mathrm{dl}$ y 2 horas mayor o igual a $153 \mathrm{mg} / \mathrm{dl}$. La CTOG en el grupo 1 fue realizada en los laboratorios clínicos de preferencia de las pacientes, la CTOG $75 \mathrm{~g}-2 \mathrm{~h}$ en el grupo 2 fue realizada en el laboratorio central del INPer. A las mujeres del grupo 2 con diagnóstico de DMG se les determinó la hemoglobina glucosilada para descartar diabetes pregestacional.

\section{Análisis estadístico}

Se realizó un análisis estadístico con el programa SPSS $^{\circledast}$ (Chicago, Illinois, EE.UU.) v. 24. Se utilizó la prueba $t$ de Student para comparar las medias para variables continuas y la prueba de chi cuadrada 0 prueba exacta de Fisher para diferencias entre proporciones. Se realizó cálculo de riesgo relativo (RR) con intervalo de confianza del 95\% (IC 95\%) para comparar la incidencia de DMG entre grupos.

\section{Resultados}

Se incluyeron un total de 144 mujeres (grupo 1, $n=48$, y grupo $2, n=96$ ). Las características basales de las mujeres se muestran en la tabla 1 . No hubo diferencias significativas en edad, peso, talla, IMC, número de gestaciones y número de factores de riesgo para desarrollar DMG. Las SDG al ingreso a control prenatal fue significativamente mayor en el grupo 2. Las participantes en el grupo 2 no tenían CTOG $75 \mathrm{~g}-2 \mathrm{~h}$ basal.

En la tabla 2 se muestran los factores de riesgo para desarrollar DMG en ambos grupos. El grupo étnico latino estuvo presente en el $100 \%$ de las participantes en ambos grupos, los factores de riesgo más frecuentes en ambos grupos fueron sedentarismo, familiar en primer grado con diabetes mellitus tipo 2, sobrepeso y obesidad. Un alto porcentaje de las participantes tenía sobrepeso u obesidad (87.5 vs. $85.7 \%$ en el grupo 1 vs. 2, respectivamente). Así mismo, más de la mitad de las participantes presentaba cinco o más factores de riesgo para desarrollar DMG; un $54.2 \%$ en el grupo 1 y un $51 \%$ en el grupo 
Tabla 1. Características maternas basales en mujeres suplementadas con mioinositol más probióticos vs. grupo control

\begin{tabular}{|c|c|c|c|}
\hline Característica & $\begin{array}{c}\text { Grupo } 1(n=48) \text { Mioinositol más } \\
\text { probióticos }\end{array}$ & $\begin{array}{c}\text { Grupo } 2(n=96) \text { Control sin } \\
\text { suplementación }\end{array}$ & $\mathbf{p}^{*}$ \\
\hline Edad (años) & $31 \pm 8.4$ & $31.1 \pm 7.4$ & 0.91 \\
\hline Peso pregestacional (kg) & $75.2 \pm 17.1$ & $75.3 \pm 16.2$ & 0.96 \\
\hline Talla (m) & $1.58 \pm .05$ & $1.56 \pm 0.06$ & 0.14 \\
\hline $\mathrm{IMC}\left(\mathrm{kg} / \mathrm{m}^{2}\right)$ & $29.9 \pm 5.6$ & $30.5 \pm 6.2$ & 0.57 \\
\hline Número de gestaciones & $2.7 \pm 2$ & $2.4 \pm 1.3$ & 0.38 \\
\hline Numero de factores de riesgo para DMG & $4.8 \pm 1.3$ & $4.6 \pm 0.98$ & 0.23 \\
\hline SDG ingreso a control prenatal & $12.0 \pm 2.2$ & $19.3 \pm 6.1$ & 0.0001 \\
\hline SDG de la CTOG inicial & $12.3 \pm 2.4$ & & \\
\hline Glucosa en ayuno (CTOG $75 \mathrm{~g}$ ) & $86.4 \pm 10.5$ & - & \\
\hline Glucosa 1 hora (CTOG 75 g) & $142.8 \pm 33.7$ & - & \\
\hline Glucosa 2 horas (CTOG $75 \mathrm{~g}$ ) & $122.3 \pm 27.5$ & - & \\
\hline
\end{tabular}

2. No hubo diferencias significativas en los factores de riesgo entre los grupos.

La incidencia de DMG en el grupo 1 fue $n=14$ (29.2\%) y en el grupo $2 \mathrm{n}=46$ (47.9\%) (RR: 0.61; IC 95\%: 0.37-0.99; $p=0.03)$. Con una reducción del RR del $39 \%$ (IC 95\%: 1-63\%) y un número necesario a tratar de 5 (IC 95\%: 59-3).

En la tabla 3 se muestran los resultados de la CTOG $75 \mathrm{~g}-2 \mathrm{~h}$; se observa que la media de glucosa en ayuno fue significativamente mayor en el grupo 2 vs. el grupo $1(p=0.01)$. No hubo diferencias en las SDG al momento de realizar la CTOG, ni en la concentración de glucosa a la hora y dos horas.

En la tabla 4 se muestra el número de valores alterados en la CTOG $75 \mathrm{~g}-2 \mathrm{~h}$. La glucosa en ayuno fue el valor alterado con mayor frecuencia 10 [20.8\%] vs. 39 [40.6\%]; $p=0.01$ para el grupo 1 vs. el 2, respectivamente. La mayoría de las mujeres con diagnóstico de DMG solo tuvieron un valor alterado en la CTOG $75 \mathrm{~g}-2 \mathrm{~h}(\mathrm{n}=7 / 48[14.6 \%]$ y $\mathrm{n}=33 / 96[33 \%])$ en el grupo 1 y 2 , respectivamente; $p=0.02$. No hubo diferencias entre los grupos con dos y tres valores alterados en la CTOG $75 \mathrm{~g}-2 \mathrm{~h}$ para el diagnóstico de DMG.

El resultado de hemoglobina glucosilada en las mujeres del grupo 2 con diagnóstico de DMG fue 5.4 $\pm 0.33 \%$ con un mínimo y máximo de 4.6 y $6.4 \%$, lo que descartó diabetes mellitus pregestacional.

\section{Discusión}

El presente estudio mostró una disminución significativa en la incidencia de DMG en un grupo de mujeres mexicanas suplementadas con mioinositol $2 \mathrm{~g}$ más $B$. lactis y $L$. rhamnosus $5 \times 10^{8}$ UFC, un sobre cada 12 horas desde las 12-14 hasta las 28 SDG. A la fecha es el primer reporte sobre el efecto de la combinación de mioinositol más probióticos para prevención de DMG en mujeres con alto riesgo de desarrollar DMG.

En el presente estudio más del $50 \%$ de las participantes presentaba cinco o más factores de riesgo, lo cual podría explicar la alta incidencia de DMG en el grupo sin suplementación (47.9\%). En ECA donde se ha evaluado la efectividad del mioinositol en la prevención de DMG, utilizando los mismos criterios diagnósticos de la Asociación internacional de grupos de estudio de diabetes y embarazo (IADPSG), la incidencia DMG en el grupo control sin suplementación fue reportada en dos estudios donde se incluyeron mujeres cuyo factor de riesgo para desarrollar DMG fue tener un familiar en primer grado con diabetes mellitus tipo 2 , en población irlandesa ${ }^{17} 18 \%$ y en población italiana $^{18} 15.3 \%$, mucho menor a lo reportado en el presente estudio. Por el contrario, la mayor incidencia de DMG en el grupo control sin suplementación con mioinositol fue del $71 \%$ en población italiana ${ }^{19}$ que tenía como factor de riesgo para desarrollar DMG una 
Tabla 2. Factores de riesgo para desarrollar DMG en mujeres suplementadas con mioinositol más probióticos vs. grupo control ${ }^{\star}$

\begin{tabular}{|c|c|c|c|}
\hline Factor de riesgo & $\begin{array}{c}\text { Grupo } 1(n=48) \text { Mioinositol más } \\
\text { probióticos }\end{array}$ & $\begin{array}{l}\text { Grupo } 2(n=96) \text { Control sin } \\
\text { suplementación }\end{array}$ & $\mathrm{p}$ \\
\hline Sobrepeso (IMC 25-29.9 kg/m²) & $22(45.8)$ & $42(43.8)$ & 0.96 \\
\hline Obesidad (IMC $\geq 30$ kg/m²) & $20(41.7)$ & $42(43.8)$ & 0.96 \\
\hline Sedentarismo & $38(79.2)$ & $83(86.5)$ & 0.26 \\
\hline Familiar en primer grado con DMT2 & 38 (79.2) & $64(66.7)$ & 0.12 \\
\hline Antecedente de DMG & $4(8.3)$ & $6(6.3)$ & 0.64 \\
\hline Antecedente de RN macrosómico & $10(20.8)$ & $10(10.4)$ & 0.09 \\
\hline Prediabetes & $9(18.8)$ & $15(15.6)$ & 0.63 \\
\hline Hipertensión arterial crónica & $6(12.5)$ & $23(24)$ & 0.11 \\
\hline Dislipidemia & $6(12.5)$ & $11(11.5)$ & 0.34 \\
\hline Antecedente de SOP & $14(29.2)$ & $16(16.7)$ & 0.08 \\
\hline
\end{tabular}

${ }^{*}$ Chi cuadrada, valores expresados como frecuencia y (\%).

IMC: índice de masa corporal; SDG: semanas de gestación; CTOG: curva de tolerancia oral a la glucosa; DMG: diabetes mellitus gestacional; DMT2: diabetes mellitus tipo 2; RN: recién nacido: SOP: síndrome de ovarios poliquísticos.

Tabla 3. Resultados de la curva de tolerancia oral a la glucosa (CTOG) 75 g-2 h en mujeres suplementadas con mioinositol más probióticos vs. grupo control

\begin{tabular}{l|c|c|c|}
\hline Característica & $\begin{array}{c}\text { Grupo 1 }(\mathrm{n}=48) \\
\text { Mioinositol más } \\
\text { probióticos }\end{array}$ & $\begin{array}{c}\text { Grupo 2 }(\mathrm{n}=96) \\
\text { Control sin } \\
\text { suplementación }\end{array}$ & $p^{*}$ \\
\hline SDG al realizar CTOG & $26.6 \pm 2.3$ & $26.2 \pm 2.4$ & 0.29 \\
\hline Glucosa en ayuno & $84.5 \pm 7.8$ & $90.3 \pm 10.3$ & 0.001 \\
\hline \begin{tabular}{l} 
Glucosa 1 hora \\
\hline Glucosa 2 horas
\end{tabular} & $137.3 \pm 29.0$ & $141.3 \pm 39.1$ & 0.53 \\
\hline $\begin{array}{l}\text { *t de Student. } \\
\text { SDG: semanas de gestación; CTOG: curva de tolerancia oral a la glucosa. }\end{array}$ & \\
\hline
\end{tabular}

Tabla 4. Número de valores alterados en la curva de tolerancia oral a la glucosa (CTOG) mujeres suplementadas con mioinositol más probióticos vs. grupo control*

\begin{tabular}{|c|c|c|c|}
\hline $\begin{array}{l}\text { Valores alterados } \\
\text { en la CTOG } 75 \text { g-2 h }\end{array}$ & $\begin{array}{c}\text { Grupo } 1(n=48) \\
\text { Mioinositol más } \\
\text { probióticos }\end{array}$ & $\begin{array}{l}\text { Grupo } 2(n=96) \\
\text { Control sin } \\
\text { suplementación }\end{array}$ & p \\
\hline Ayuno & $10(20.8)$ & $39(40.6)$ & 0.01 \\
\hline 1 hora & $6(12.5)$ & $16(16.7)$ & 0.51 \\
\hline 2 horas & $7(14.6)$ & $14(14.6)$ & 1 \\
\hline $\begin{array}{l}\text { Para el diagnóstico } \\
\text { de DMG }\end{array}$ & $14(29.2)$ & $46(46.9)$ & 0.03 \\
\hline Uno & $7(14.6)$ & 32 (33.3) & 0.02 \\
\hline Dos & $5(10.4)$ & $5(5.2)$ & 0.42 \\
\hline Tres & $2(4.2)$ & $9(9.4)$ & 0.44 \\
\hline
\end{tabular}

glucosa de ayuno entre 92 y $125 \mathrm{mg} / \mathrm{dl}$, una incidencia mayor a la reportada en el presente estudio.

La disminución en la incidencia de DMG en el grupo suplementado con mioinositol más probióticos fue del $39 \%$, lo cual es menor a lo reportado en $\mathrm{ECA}^{18-21}$ que han utilizado $4 \mathrm{~g}$ de mioinositol y han mostrado una disminución en la incidencia de DMG del 52 al 92\%, lo que podría explicarse por las diferencias en el grupo étnico y el mayor número de factores de riesgo en el presente estudio.

En relación con los probióticos, solo en un $\mathrm{ECA}^{15}$, realizado en Irlanda, ha utilizado $B$. lactis y $L$. rhamnosus $1 \times 10^{10} \mathrm{UFC} /$ día, administrados desde la semana 12 de gestación hasta el término del embarazo. La incidencia de DMG reportada en el grupo control fue del 34 vs. el $13 \%$ en el grupo con dieta más probióticos; todas las participantes fueron caucásicas, sanas y con un IMC promedio de $23.6 \pm 3.8 \mathrm{~kg} / \mathrm{m}^{2}$ y no se utilizaron los criterios de IADPSG para el diagnóstico de DMG, lo cual podría explicar la menor incidencia de DMG en el grupo control comparado con el presente estudio.

En relación con la frecuencia de valores alterados, la glucosa de ayuno fue el valor alterado con mayor frecuencia en el grupo control comparado con el grupo que recibió mioinositol y probióticos, lo cual se explica por el efecto del mioinositol a dosis de $4 \mathrm{~g} /$ día para disminuir significativamente la glucosa de ayuno y que no se ha observado con mioinositol a dosis de $1.1 \mathrm{~g} /$ día $^{22}$. Así mismo, se ha reportado un efecto 
significativo sobre la disminución de la glucosa de ayuno y la resistencia a la insulina con el uso de probióticos durante el embarazo utilizado diferentes probióticos, incluyendo $B$. lactis y $L$. rhamnosus con intervenciones desde 4 hasta 12 semanas $^{23}$.

El presente estudio tiene ciertas limitaciones, entre ellas el tamaño de muestra es limitado, no se reportan otros desenlaces perinatales (como nacimiento pretérmino, preeclampsia y peso neonatal), los grupos de estudio no llevaron el control prenatal en el mismo periodo de tiempo, el grupo control no tenía una CTOG $75 \mathrm{~g}-2 \mathrm{~h}$ basal y las SDG al inicio del control prenatal fueron significativamente mayores en el grupo control. Tampoco se reporta si además de la suplementación las participantes realizaron algún tipo de dieta y/o ejercicio, ni la ganancia de peso gestacional, que podrían influir en la incidencia de DMG. Si bien el efecto de la dieta y el ejercicio han mostrado un beneficio limitado en la prevención de DMG, por los diferentes tipos de intervención y limitaciones para la implementación y adherencia, se ha reportado que las mujeres que inician cambios en el estilo de vida antes de las 15 SDG y que logran un adecuado control de la ganancia de peso gestacional pueden disminuir la incidencia de DMG en un 15 a $18 \% \%^{24}$.

Dentro de las fortalezas del estudio podemos mencionar que es un grupo de mujeres con tres o más factores de riesgo para DMG, que el grupo control fue pareado por edad e IMC y que el objetivo primario del estudio fue evaluar la incidencia del DMG. Si bien las mujeres del grupo control no contaban con una CTOG $75 \mathrm{~g}-2 \mathrm{~h}$ antes de las 15 SDG para descartar diabetes pregestacional, sí fue posible descartarla con la medición de hemoglobina glucosilada. Si bien la combinación de mioinositol más probióticos mostró un efecto benéfico en la prevención de DMG, se requieren $E C A$, multicéntricos y con mayor tamaño de muestra, para corroborar los hallazgos del presente estudio.

\section{Conclusiones}

La suplementación con mioinositol más probióticos desde las 12-14 SDG disminuye la incidencia de DMG en mujeres mexicanas. Se requieren ECA para confirmar dichos hallazgos.

\section{Financiamiento}

El presente estudio recibió financiamiento de Nestlé México.

\section{Conflicto de intereses}

Enrique Reyes-Muñoz y Salvador Espino y Sosa son speakers e integrantes del Expert Team de Nestlé México. El resto de los autores no tienen conflictos de intereses.

\section{Responsabilidades éticas}

Protección de personas y animales. Los autores declaran que para esta investigación no se han realizado experimentos en seres humanos ni en animales.

Confidencialidad de los datos. Los autores declaran que han seguido los protocolos de su centro de trabajo sobre la publicación de datos de pacientes.

Derecho a la privacidad y consentimiento informado. Los autores declaran que en este artículo no aparecen datos de pacientes.

\section{Bibliografía}

1. American Diabetes Association. 2. Classification and diagnosis of diabetes: Standards of Medical Care in Diabetes-2020. Diabetes Care. 2020;43:S14-S31.

2. Behboudi-Gandevani S, Amiri M, Yarandi RB, Tehrani FR. The impact of diagnostic criteria for gestational diabetes on its prevalence: a systematic review and meta-analysis. Diabetol Metab Syndr. 2019;11:11.

3. Nguyen CL, Pham NM, Binns CW, Van Duong D, Lee AH. Prevalence of gestational diabetes mellitus in Eastern and Southeastern Asia: A systematic review and meta-analysis. J Diabetes Res. 2018;2018:1-10.

4. Diabetes Atlas 2019 [Internet]. International Diabetes Federation [Consultado en junio de 2020]. Disponible en: https://www.diabetesatlas.org/ upload/resources/material/20200302 133351 IDFATLAS9e-final-web.pdf

5. Dainelli L, Prieto-Patron A, Silva-Zolezzi I, Sosa-Rubi SG, Espino y Sosa S, Reyes-Muñoz E et al. Screening and management of gestational diabetes in Mexico: results from a survey of multilocation, multi-health care institution practitioners. Diabetes Metab Syndr Obes. 2018;11: 05-16.

6. Hartling L, Dryden DM, Guthrie A, Muise M, Vandermeer B, Donovan L. Benefits and harms of treating gestational diabetes mellitus: a systematic review and meta-analysis for the U.S. Preventive Services Task Force and the National Institutes of Health Office of Medical Applications of Research. Ann Intern Med. 2013;159:123-9.

7. HAPO Study Cooperative Research Group, Metzger BE, Lowe LP, Dyer AR, Trimble ER, Chaovarindr U, et al. Hyperglycemia and adverse pregnancy outcomes. N Engl J Med. 2008;358:1991-2002.

8. Plows JF, Stanley JL, Baker PN, Reynolds CM, Vickers MH. The pathophysiology of gestational diabetes mellitus. Int J Mol Sci. 2018:19(11):3342.

9. Agha-Jaffar R, Oliver N, Johnston D, Robinson S. Gestational diabetes mellitus: does an effective prevention strategy exist? Nat Rev Endocrinol. 2016:12:533-46.

10. American College of Obstetricians and Gynecologists. Committee on Practice Bulletins-Obstetrics. ACOG Practice Bulletin Number 190: Gestational Diabetes Mellitus. Obstet Gynecol. 2018;131:e49-e64.

11. Saltiel AR. Second messengers of insulin action. Biology of Cellular Transducing Signals. 1990;391-399.

12. Corrado F, D'Anna R, Di Vieste G, Giordano D, Pintaudi B, Santamaria A, et al. The effect of myoinositol supplementation on insulin resistance in patients with gestational diabetes. Diabet Med. 2011;28:972-5.

13. Zhang H, Lv Y, Li Z, Sun L, Guo W. The efficacy of myo-inositol supplementation to prevent gestational diabetes onset: a meta-analysis of randomized controlled trials. J Matern Fetal Neonatal Med. 2019; 32:2249-55

14. Gomez-Arango LF, Barrett HL, McIntyre HD, Callaway LK, Morrison M, Dekker Nitert $\mathrm{M}$, et al. Connections between the gut microbiome and metabolic hormones in early pregnancy in overweight and obese women. Diabetes. 2016;65:2214-23.

15. Luoto R, Laitinen K, Nermes M, Isolauri $\mathrm{E}$. Impact of maternal probiotic-supplemented dietary counselling on pregnancy outcome and prenatal and postnatal growth: a double-blind, placebo-controlled study. $\mathrm{Br} \mathrm{J}$ Nutr. 2010;103:1792-9 
16. Wickens KL, Barthow CA, Murphy R, Abels PR, Maude RM, Stone PR, et al. Early pregnancy probiotic supplementation with Lactobacillus rhamnosus HN001 may reduce the prevalence of gestational diabetes mellitus: a randomised controlled trial. Br J Nutr. 2017;117:804-13.

17. Farren M, Daly N, McKeating A, Kinsley B, Turner MJ, Daly S. The prevention of gestational diabetes mellitus with antenatal oral inositol supplementation: A randomized controlled trial. Diabetes Care. 2017;40:759-63.

18. D'Anna R, Scilipoti A, Giordano D, Caruso C, Cannata ML, Interdonato ML, et al. Myo-inositol supplementation and onset of gestational diabetes mellitus in pregnant women with a family history of type 2 diabetes: a prospective, randomized, placebo-controlled study. Diabetes Care. 2013;36:854-7.

19. Matarrelli B, Vitacolonna E, D'Angelo M, Pavone G, Mattei PA, Liberati $\mathrm{M}$, et al. Effect of dietary myo-inositol supplementation in pregnancy on the incidence of maternal gestational diabetes mellitus and fetal outcomes: a randomized controlled trial. J Matern Fetal Neonatal Med. 2013;26:967-72.
20. D’Anna R, Di Benedetto A, Scilipoti A, Santamaria A, Interdonato ML, Petrella $\mathrm{E}$, et al. Myo-inositol supplementation for prevention of gestational diabetes in obese pregnant women: A randomized controlled trial. Obstet Gynecol. 2015;126:310-5.

21. Santamaria A, Di Benedetto A, Petrella E, Pintaudi B, Corrado F, D'Anna R, et al. Myo-inositol may prevent gestational diabetes onset in overweight women: a randomized, controlled trial. J Matern Fetal Neonatal Med. 2016;29:3234-7.

22. Vitagliano A, Saccone G, Cosmi E Visentin S, Dessole F, Ambrosini G, et al. Inositol for the prevention of gestational diabetes: a systematic review and meta-analysis of randomized controlled trials. Arch Gynecol Obstet. 2019;299:55-68

23. Han M-M, Sun J-F, Su X-H, Peng Y-F, Goyal H, Wu C-H, et al. Probiotics improve glucose and lipid metabolism in pregnant women: a meta-analysis. Ann Transl Med. 2019;7:99.

24. Egan AM, Simmons D. Lessons learned from lifestyle prevention trials in gestational diabetes mellitus. Diabet Med. 2019;36:142-50. 